\title{
Direct observation of negative refraction at the millimeter-wave regime by using a flat composite metamaterial
}

\author{
Kamil Boratay Alici* and Ekmel Ozbay \\ Nanotechnology Research Center, Department of Physics, Department of Electrical and Electronics Engineering, \\ Bilkent University, Bilkent, 06800 Ankara, Turkey \\ *Corresponding author: bora@fen.bilkent.edu.tr
}

Received January 9, 2009; revised June 2, 2009; accepted July 19, 2009; posted July 28, 2009 (Doc. ID 105911); published August 14, 2009

\begin{abstract}
We studied the transmission characteristics of a one-dimensional metamaterial slab operating at the millimeter-wave regime. The double-negative nature was proven by using a normal incidence transmission study. A split-ring resonator based metamaterial flat lens with five layers at the propagation direction was constructed and illuminated with oblique incidence. From the scanning experiments, the shifting of the beam to the negative side was observed. The experimental results are in agreement with the Drude-Lorentz model based simulations. Two-dimensional field maps that were obtained from these simulations demonstrated negative refraction, negative phase velocity, and the reflected beam properties at $99 \mathrm{GHz}$. (C) 2009 Optical Society of America

OCIS codes: $160.3918,160.4670,260.5740$.
\end{abstract}

The macroscopic behavior of a homogeneous medium can simply be specified by using electric permittivity $(\epsilon)$ and magnetic permeability $(\mu)$ parameters. In nature, materials do not exhibit simultaneous negative $\mu$ and $\epsilon$. In recent years, it has been demonstrated that artificial periodic structures, with optimized unit cell architectures, form an effective medium with assigned effective $\epsilon$ and $\mu$, which can both be negative [1]. The medium was called metamaterial, and it has exciting properties such as a negative index of refraction, negative phase velocity, backward Cherenkov radiation, and the reversal of the Doppler shift [2]. A widespread method for the realization of negative-index metamaterials is to utilize the magnetic resonance of subwavelength structures for negative- $\mu$ [3] and low-frequency plasmonic systems composed of continuous thin wires for negative $\epsilon[4,5]$.

Metamaterials can be incorporated into devices that operate at any band of the electromagnetic spectrum from microwave to infrared and visible regimes. There is a potential for improving their current device performances and also for creating novel device properties. For example, Wiltshire et al. demonstrated that the introduction of $\mu$-negative materials can improve the image quality in magnetic resonance imaging by guiding the radio frequency flux from an object to the receiver [6]. Bilotti et al. designed an efficient $\mu$-negative material loaded patch antenna whose electrical size is much smaller than the conventional case [7]. On the other hand, the major trend is to demonstrate the metamaterial concepts at higher frequencies. Operation frequencies of $5 \mathrm{GHz}$ [1], $100 \mathrm{GHz}$ [8,9], $5 \mathrm{THz}$ [10], $100 \mathrm{THz}$ [11], and $370 \mathrm{THz}$ [12] are demonstrated by scaling the unit cell dimensions and by using various fabrication techniques. However, due to the saturating magnetic response of $\mu$-negative medium ele- ments [13], it is very difficult to demonstrate a doublenegative metamaterial medium operating at the visible band. The demonstration of a superlens at the visible band, whose imaging properties are not limited by diffraction, remains to be an attractive research field. On the other hand, metamaterial applications are not limited to the optical regime; there is currently ongoing research at the terahertz and gigahertz regimes of the electromagnetic spectrum. For the experimental verification of double negativity at different frequency bands, different fabrication and measurement techniques should be applied. For example, while printed circuit board technologies are adequate to obtain double-negative operation at $3 \mathrm{GHz}$, optical lithography and clean room facilities are needed for $300 \mathrm{GHz}$. Similarly, at $3 \mathrm{GHz}$, horn antennas are used as the radiation sources and sinks while, at the infrared regime, we utilize light bulbs and InGaAs detectors. There can be unique device applications specific to a frequency band due to the properties of the materials used for the device realization and atmospheric conditions. The experimental study of metamaterials at frequency bands other than the optical regime, such as terahertz and gigahertz frequencies, has its own significance and $100 \mathrm{GHz}$ could be thought of as the starting point of terahertz applications, in which it plays the role of a bridge between the microwave and terahertz regimes. In the present study, we verify the negative refraction property of a rectangular slab metamaterial operating at $100 \mathrm{GHz}$ by scanning the transmitted beam at the second metamaterial-air interface.

The paper is organized as follows: after a description of the designed metamaterial, we prove that the metamaterial has simultaneously negative $\epsilon$ and $\mu$ by using the qualitative effective medium theory methods. We briefly 
explain our fabrication, measurement, and numerical calculation methods. And finally, we demonstrate the results of the Gaussian beam shifting method [14] at the second metamaterial-air interface.

Our design was composed of split-ring resonators (SRRs) and continuous wires. A schematic of the periodic configuration and unit cell are shown in Fig. 1 . The SRR parameters are as follows: the strip width $w=55 \mu \mathrm{m}$, separation between the strips $s=55 \mu \mathrm{m}$, split width $g$ $=55 \mu \mathrm{m}$, and resonator side length $l=440 \mu \mathrm{m}$. The continuous wires of width $u=275 \mu \mathrm{m}$ are situated on the back face of the substrate and are shown in Fig. 1 (right). The period was $a_{z}=550 \mu \mathrm{m}$ in the propagation direction $(-z), a_{y}=550 \mu \mathrm{m}$ in the $y$ direction, and $a_{x} \sim 400 \mu \mathrm{m}$ in the $x$ direction. There are five or three layers in the $z$ direction, 45 layers in the $y$ direction, and 54 layers in the $x$ direction. The substrate is Rogers RT/duroid 5880 with a dielectric constant $\epsilon=2.0$ and a dissipation factor $\tan \delta$ $=0.0009$. The substrate thickness was $t=254 \mu \mathrm{m}$, and the deposited copper thickness was $h=9 \mu \mathrm{m}$. The metamaterial layers were illuminated with a linearly polarized wave propagating at the $-z$ direction, the E-field was in the $y$ direction, and the $\mathrm{H}$-field was in the $x$ direction.

In our simulations, we used the commercial fullwave code, CST Microwave Studio, which is based on the finite integration technique [15]. The metamaterial unit cell was inserted into the simulation domain, and periodic boundary conditions were applied at the lateral directions ( $y$ and $x$ directions). The transmission data was obtained via point probes.

We demonstrate the results of the transmission-based qualitative effective medium theory analysis in Fig. 2. This analysis proves whether the designed medium possesses simultaneously negative effective permeability and permittivity [9,16-18]. In this analysis, first we consider the transmission response of the SRR medium and its shortened version (sh-SRR), in which the split width $g$ $=0$ is equal to zero. The shortened SRR is also known as closed ring resonator (CRR). In Fig. 2 on the left-hand side, we showed the simulation and experiment results of the first part. The disappearance of the stop-band in the transmission data of the sh-SRR medium implies that the resonance of the SRR medium is magnetically originated. Thereby, at around the resonance frequency, the effective permeability was negative $(\mu<0)$. Second, we checked the transmission data of the composite metamaterial (CMM) that was shown in Fig. 2, right-hand side. At around the

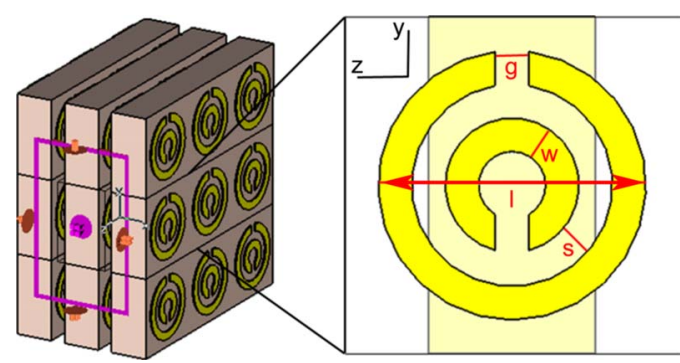

Fig. 1. (Color online) Schematic of the designed split-ring resonator based metamaterial medium. Periodic configuration and SRR parameters are shown: $\mathrm{g}=\mathrm{w}=\mathrm{s}=55 \mu \mathrm{m}, \mathrm{l}=440 \mu \mathrm{m}$. The propagation direction was $-z$ direction, E-field was in the $y$ direction and the $\mathrm{H}$-field was in the $x$ direction.

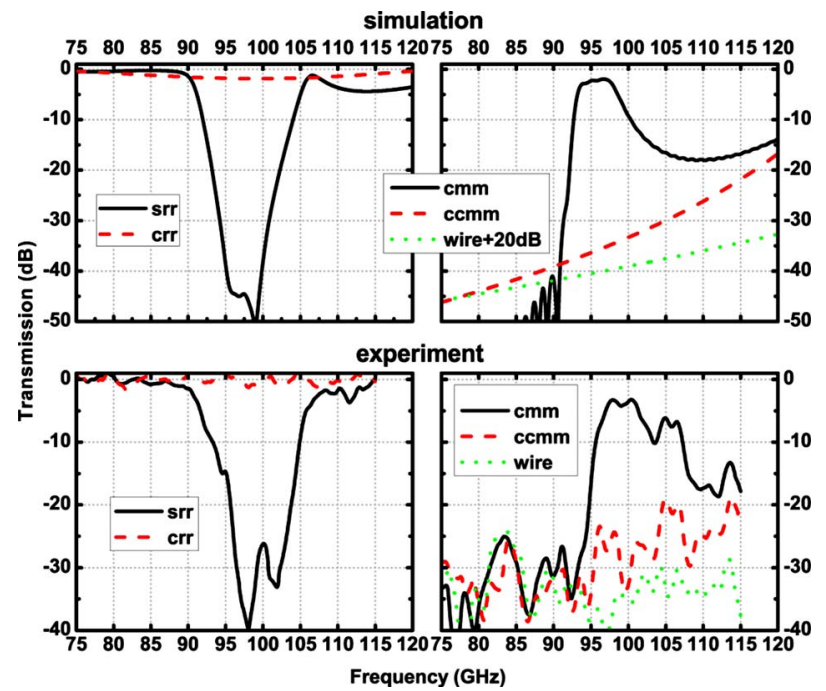

Fig. 2. (Color online) Transmission spectrum for three layered metamaterials in the propagation direction. (Left) The SRR (solid curve) and CRR (dashed curve). (Right) The CMM (solid curve) and shortened CMM (dashed curve), i.e., closed composite metamaterial (CCMM).

magnetic resonance frequency, we saw a transmission peak, which was expected to be due to the double negativity of the medium. We decided whether the permittivity of the CMM medium was negative by considering its shortened version (sh-CMM). Their plasma responses were nearly the same. The sh-CMM medium was composed of continuous wires and sh-SRRs, and its plasma frequency was around $150 \mathrm{GHz}$. Below the plasma frequency, the permittivity was negative and transmission was forbidden, as shown in Fig. 2.

At this point, we would like to discuss one of the significant differences between the previously demonstrated $100 \mathrm{GHz}$ metamaterial of our group [8] and the one presented in this paper. One of the critical parameters for the metamaterial study is the losses. In [8] we used glass as substrate and the metal thickness was close to the skin depth at $100 \mathrm{GHz}$. Both the use of glass material and thin metal layer made that metamaterial very lossy and the scaled transmission peak for normal incidence was $\sim-25 \mathrm{~dB}$. This lossy nature prohibited us from the demonstration of beam shifting, metamaterial prism, and farfield effects at the millimeter wave scale. The losses are the main obstacle for obtaining metamaterials composed of deep subwavelength elements at the optical and visible regimes of the electromagnetic spectrum. In this study, we demonstrated a low loss metamaterial with scaled transmission peak for normal incidence of $\sim-2 \mathrm{~dB}$. By using this metamaterial beam shifting, metamaterial prism and far-field effects experiments can be realized rather easily.

At $100 \mathrm{GHz}$ the minimum dimensions of the metamaterial elements were $\sim 50 \mu \mathrm{m}$ and the unit cell dimensions were about a half millimeter. Compared to the $3 \mathrm{GHz}$ ones [14] these structures were much more difficult to fabricate and handle. The measurements at $100 \mathrm{GHz}$ were not performed with standard network analyzers, as they are extremely expensive [19]. We constructed a homemade millimeter-wave network analyzer 
by adding several instruments to an HP8510C network analyzer.

The schematic of the refraction spectra measurement setup is shown in Fig. 3(a). The setup consists of a millimeter-wave network analyzer, automated linear translation system, and two horn antennas as the transmitter and receiver. There were five metamaterial layers in the propagation direction. The transmitter horn antenna was on the right-hand side of the metamaterial with respect to its central axis. We scanned the spatial intensity distribution along the second metamaterial-air interface from 75 to $115 \mathrm{GHz}$. In Fig. 3(b) we made an additional analysis related to anisotropy in order to clarify that this anisotropic metamaterial has a negative index that indeed leads to the negative beam shift. In this analysis, we used the retrieval formalism developed in [20], which takes into account the asymmetry of the medium at the propagation direction. Even though the method of [20] was developed by considering a homogeneous media, it can be used to retrieve the effective medium parameters of anisotropic multidimensional metamaterials [20]. From the complex scattering parameters we extracted the metamaterial's refractive index and, as shown in Fig. 3(b), we demonstrated that for oblique incidence with angle used in the experiment $\left(22^{\circ}\right)$ the metamaterial have a negative index of refraction at around $100 \mathrm{GHz}$. The refractive index $n$ was -1.0 at $99 \mathrm{GHz}$. Recently an experimental proof on the insensitivity of SRR-based metamaterials to the angle of incidence was provided. They are in parallel with the analyses' results given here [21].

Figure 4 shows the transmission spectra as a function of frequency, in which the lateral position for the angle of incidence was $\alpha=22^{\circ}$. For control purposes, we also measured the free-space response. It is evident that, for the metamaterial case, the transmitted beam appeared on the right-hand side. Thereby, the index of refraction of the metamaterial medium was negative. The frequency cuts at $99 \mathrm{GHz}$ are shown in Fig. 5, in which the appearance of

(a)

\section{Receiver: horn antenna}
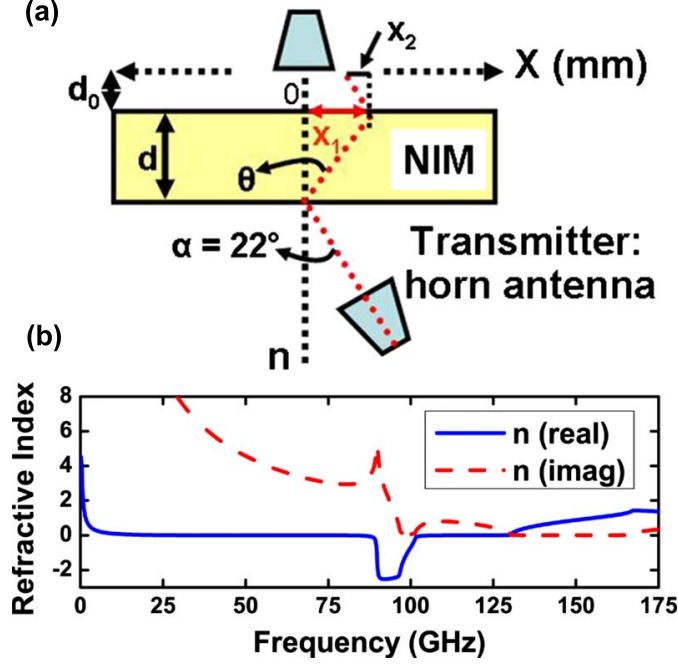

Fig. 3. (Color online) (a) Beam-shifting experiment geometry. (b) Retrieved effective refractive index for the oblique incidence for $\alpha=22^{\circ}$.

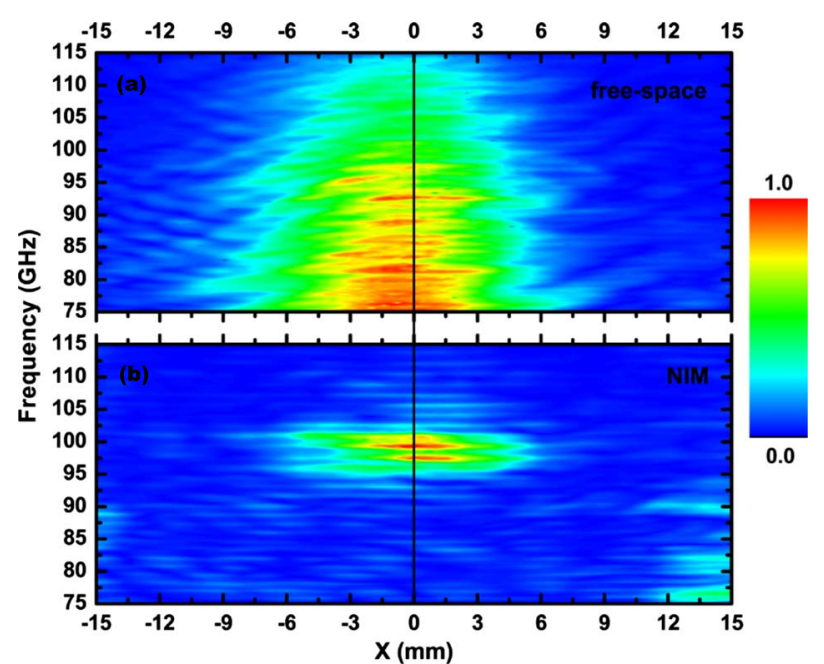

Fig. 4. (Color online) Transmission spectra as a function of frequency and scanning distance. (a) Free space, (b) negative index metamaterial.

a negatively refracted beam can be seen more clearly. By using Snell's law of refraction, we determined the refractive index from the experiment. The parameters shown in Fig. 3(a) are as follows: thickness of the slab was $d$ $=2.75 \mathrm{~mm}$, distance of the horn antenna to the interface was $d_{0}=1 \mathrm{~mm}$, measured shift distance was $x=0.6 \mathrm{~mm}$, and the incidence angle was $\alpha=22^{\circ}$. By using simple geometric equations and Snell's law: $n_{\text {air }} \sin \alpha=n_{\mathrm{NIM}} \sin (-\theta)$, $x_{1}=d \tan \theta, x_{2}=d_{0} \tan \alpha, x=x_{1}-x_{2}$, we derived the other parameters: refraction angle $\theta=20^{\circ}$, shift distances $x_{1}$ $=1.0 \mathrm{~mm}, x_{2}=0.4 \mathrm{~mm}$, and refractive index $n=-1.0$. The results are in good agreement with the theoretical calculations. By just looking at the beam shift we cannot claim that the medium has a negative index. Thereby, here we demonstrated that there is a correlation between the negative band region determined by the qualitative effective medium theory analysis, retrieval analysis, and the beam-shifting experiment. We do not expect such a frequency-dependent response from an arbitrary anisotropic medium.

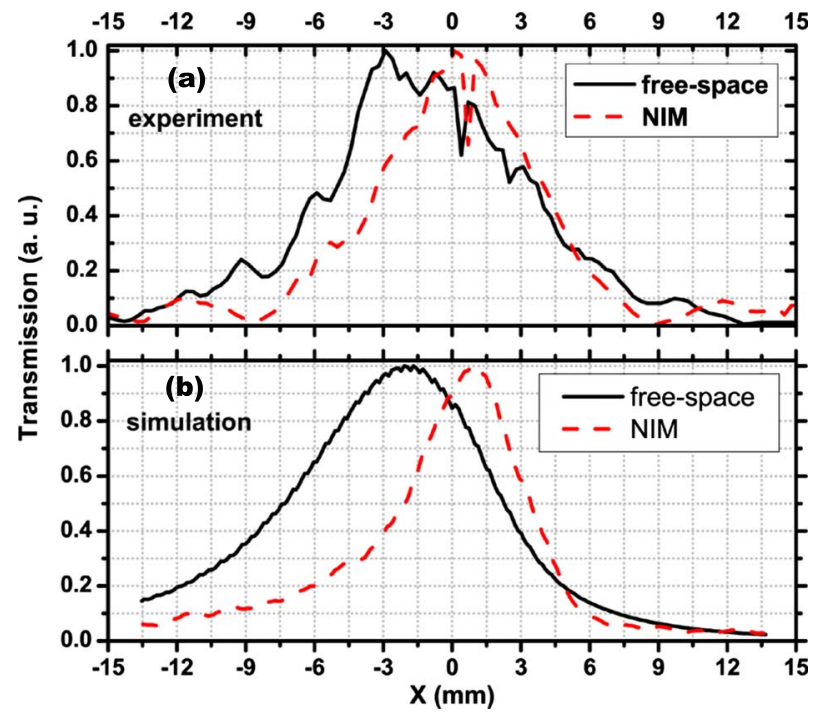

Fig. 5. (Color online) Frequency cuts at $99 \mathrm{GHz}$. (a) Experiment: free space (solid curve)-negative index metamaterial (NIM) (dashed curve). (b) Drude-Lorentz simulations. 
We also confirmed the experimental results by using CST simulations. In these simulations, we modeled our negative index medium with the isotropic Drude-Lorentz model. The Drude model formula was $\epsilon(\omega)=\epsilon_{\infty}+\omega_{p}{ }^{2} / \omega(\omega$ $\left.-i \nu_{c}\right)$ with the following parameters: plasma frequency $\omega_{p}=2 \pi 130 \mathrm{GHz}$, collision frequency $\nu_{c}=0.01 \mathrm{~Hz}$. For the magnetic dispersion, the Lorentz model formula was $\mu(\omega)=\mu_{\infty}+\left(\mu_{\mathrm{s}}-\mu_{\infty}\right) \omega_{0}{ }^{2} /\left(\omega_{0}{ }^{2}+i \omega \gamma-\omega^{2}\right)$ where the parameters permeability infinity $\mu_{\infty}=1$, permeability static $\mu_{\mathrm{S}}$ $=1.07$, resonance frequency $\omega_{0}=2 \pi 97.9 \mathrm{GHz}$ and the damping frequency $\gamma=1.3 \mathrm{GHz}$ were used. The DrudeLorentz model parameters were selected in such a way that they were in good agreement with the retrieved effective parameters of the metamaterial medium at around $99 \mathrm{GHz}$. We made a Drude-Lorentz model fit to the retrieved effective medium parameters: permittivity $(\epsilon)$ and permeability $(\mu)$ and selected the model parameters by specifically paying attention to $99 \mathrm{GHz}$. We applied perfect electric conductor boundary conditions at the vertical direction $(y)$ and perfect absorbing boundary conditions in other directions. The transmitter horn antenna model that was used in the simulations has the same physical parameters as the one used in the experiments. It was fed with a waveguide port. By this method, the field propagation and far-field profiles were simulated with efficient computation power. We demonstrated the field profile of the experiment in Fig. 6 Media 1 as an animation. The appearance of the beam on the right-hand side of the second interface, negative phase velocity inside the metamaterial medium, negative refraction, and the reflection properties can all be seen in this animation. In Fig. 6 instead of simulating the actual slab composed of many metamaterial unit cells, we used a homogeneous medium with assigned index and impedance. By this method, we were able to observe the field profile in and around the negative index metamaterial slab. The main advantage of this method was the reduction of required computer power and memory.

In summary, we confirmed by direct field scan measurements, a one-dimensional metamaterial lens that is designed to be double negative by using the qualitative effective medium theory, in which it indeed refracts the obliquely incident waves to the negative direction. The study was performed both experimentally and numerically at around $100 \mathrm{GHz}$.

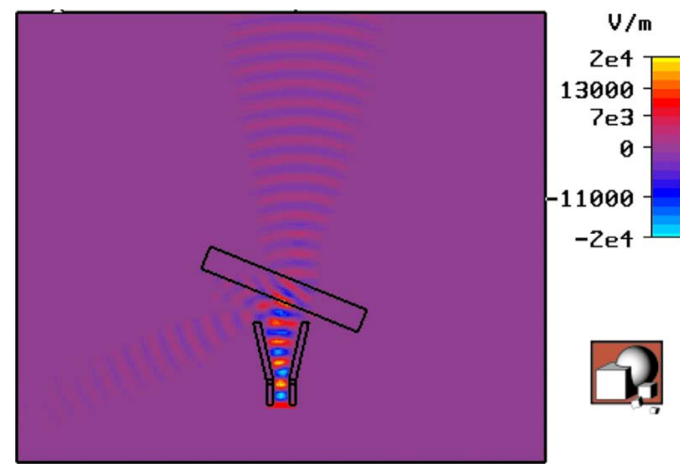

Fig. 6. (Color online) (Media 1) Electric field magnitude in $y$ direction at $99 \mathrm{GHz}$.

\section{ACKNOWLEDGMENTS}

This work is supported by the European Union under the projects EU-METAMORPHOSE, EU-PHOREMOST, EU-PHOME, and EU-ECONAM, and TUBITAK under project numbers 105E066, 105A005, 106E198, and 106A017. One of the authors (E.O.) also acknowledges partial support from the Turkish Academy of Sciences.

\section{REFERENCES}

1. D. R. Smith, W. J. Padilla, D. C. Vier, S. C. Nemat-Nasser, and S. Schultz, "Composite medium with simultaneous negative permeability and permittivity," Phys. Rev. Lett. 84, 4184-4187 (2000)

2. V. G. Veselago, "Electrodynamics of substances with simultaneously negative electrical and magnetic permeabilities," Sov. Phys. Usp. 10, 509-516 (1968).

3. J. B. Pendry, A. J. Holden, D. J. Robbins, and W. J. Stewart, "Magnetism from conductors and enhanced nonlinear phenomena," IEEE Trans. Microwave Theory Tech. 47, 2075-2084 (1999).

4. J. B. Pendry, A. J. Holden, W. J. Stewart, and I. Youngs, "Extremely low frequency plasmons in metallic mesostructures," Phys. Rev. Lett. 76, 4773-4776 (1996).

5. E. Ozbay and K. Aydin, "Negative refraction and imaging beyond the diffraction limit by a two-dimensional lefthanded metamaterial," Photonics Nanostruct. Fundam. Appl. 6, 108-115 (2008).

6. M. C. K. Wiltshire, J. B. Pendry, I. R. Young, D. J. Larkman, D. J. Gilderdale, and J. V. Hajnal, "Microstructured magnetic materials for RF flux guides in magnetic resonance imaging," Science 291, 849-851 (2001).

7. F. Bilotti, A. Alu, and L. Vegni, "Design of miniaturized metamaterial patch antennas with $\mu$-negative loading," IEEE Trans. Antennas Propag. 56, 1640-1647 (2008).

8. M. Gokkavas, K. Guven, I. Bulu, K. Aydin, R. S. Penciu, M. Kafesaki, C. M. Soukoulis, and E. Ozbay, "Experimental demonstration of a left-handed metamaterial operating at 100 GHz," Phys. Rev. B 73, 193103 (2006).

9. K. B. Alici and E. Ozbay, "Characterization and tilted response of a fishnet metamaterial operating at $100 \mathrm{GHz}$," J. Phys. D 41, 135011 (2008).

10. B. D. F. Casse, M. O. Moser, J. W. Lee, M. Bahou, S. Inglis, and L. K. Jian, "Towards three-dimensional and multilayer rod-split-ring metamaterial structures by means of deep x-ray lithography," Appl. Phys. Lett. 90, 254106 (2007).

11. S. Linden, C. Enkrich, M. Wegener, J. Zhou, Th. Koschny, and C. M. Soukoulis, "Magnetic response of metamaterials at 100 terahertz," Science 306, 1351-1353 (2004).

12. C. Enkrich, M. Wegener, S. Linden, S. Burger, L. Zschiedrich, F. Schmidt, J. F. Zhou, Th. Koschny, and C. M. Soukoulis, "Magnetic metamaterials at telecommunication and visible frequencies," Phys. Rev. Lett. 95, 203901 (2005).

13. J. Zhou, Th. Koschny, M. Kafesaki, E. N. Economou, J. B. Pendry, and C. M. Soukoulis, "Saturation of the magnetic response of split-ring resonators at optical frequencies," Phys. Rev. Lett. 95, 223902 (2005).

14. K. Aydin and E. Ozbay, "Negative refraction through an impedance matched left-handed metamaterial slab," J. Opt. Soc. Am. B 23, 415-418 (2006).

15. User Manual, Version 5.0, CST GmbH, Darmstadt, Germany, 2005, http://www.cst.de.

16. E. Ozbay, K. Aydin, E. Cubukcu, and M. Bayindir, "Transmission and reflection properties of composite double negative metamaterials in free space," IEEE Trans. Antennas Propag. 51, 2592-2595 (2003).

17. Th. Koschny, M. Kafesaki, E. N. Economou, and C. M. Soukoulis, "Effective medium theory of lefthanded materials," Phys. Rev. Lett. 93, 107402 (2004).

18. K. B. Alici and E. Ozbay, "A planar metamaterial: 
polarization independent fishnet structure," Photonics Nanostruct. Fundam. Appl. 6, 102-107 (2008).

19. http://www.home.agilent.com. Agilent N5250C MillimeterWave PNA Series vector network analyzer

20. D. R. Smith, D. C. Vier, Th. Koschny, and C. M. Soukoulis,
"Electromagnetic parameter retrieval from inhomogeneous metamaterials," Phys. Rev. E 71, 036617 (2005).

21. K. B. Alici and E. Ozbay, "Oblique response of a split-ringresonator-based left-handed metamaterial slab," Opt. Lett. 34, 2294-2296 (2009). 